\title{
Effectiveness of Remineralization Treatments on Incipient Lesions: A Controlled Clinical Trial
}

\author{
Said Karabekiroglu ${ }^{1}$, Emire Aybuke Erdur ${ }^{2}$, Zehra Ileri ${ }^{2 *}$ and Nimet Unlu ${ }^{3}$ \\ ${ }^{1}$ Department of Restorative Dentistry, Necmettin Erbakan University, Turkey \\ ${ }^{2}$ Department of Orthodontics, Selcuk University, Turkey \\ ${ }^{3}$ Department of Restorative Dentistry, Selcuk University, Turkey
}

*Corresponding author: Dr. Zehra Ileri, Depatment of Orthodontics, Selcuk University, Selçuklu-42079, Kampüs/Konya, Turkey, Tel: +90-555-309-88-92, Fax:+90-332-241-00-62, E-mail: zehra.ileri@gmail.com

\begin{abstract}
Objective: To determine the efficacy of four different methods in treating incipient lesions (ILs) during orthodontic treatment with fixed appliances.

Materials and methods: Twenty-nine subjects with a total of 218 teeth with (Diagnodent Pen) DD values $>0$ were included. Subjects were divided randomly into four groups: Control [ 7 subjects, 54 teeth]; $5 \%$ Sodium fluoride varnish (FL-V) [8 subjects, 59 teeth]; Chlorhexidine varnish: 1\% chlorhexidine diacetate and $1 \%$ thymol $(\mathrm{CHX}-\mathrm{V})[7$ subjects, 54 teeth], and $10 \%$ Casein phosphopeptide-amorphous calcium phosphate (CPP-ACP) [7 subjects, 51 teeth]. All patients used fluoride-containing toothpaste, and the control group received fluoride gel every 3 months during the first 6 months. The intervention period was the first 6 months; The total study period was 12 months. The labial surfaces of anterior teeth were assessed by use of DD and ICDAS II criteria at baseline (T0), 6-month (T1), and 12-month (T2) follow-up visits.
\end{abstract}

Results: There were no significant differences between the four groups at baseline $(p>0.05)$. Statistically significant differences were found between the mean DD readings of the CPP-ACP and other groups at T1 and T2 ( $<<0.05)$. The DD values of the ILs decreased in all groups during the intervention period (T0-T1), except for the control group. The mean DD readings increased in all groups during the follow-up period (T1-T2). The ICDAS II scores showed no statistically significant difference between all test groups at T1 and T2 ( $p>0.05)$.

Conclusions: The daily usage of CPP-ACP was as effective as other test methods for improving the appearance of ILs, and it was found to significantly increase the remineralization of subsurface lesions.

\section{Introduction}

Incipient lesions (ILS) are a common iatrogenic effect seen in patients undergoing orthodontic treatment with fixed appliances [1]. Although the labial surfaces of the teeth are generally not susceptible to caries, orthodontic brackets increase the risk of caries by increasing plaque retention in that area [2]. The overall prevalence of ILS among orthodontic patients has been reported to be between $26 \%$ and $89 \%$ [3-6]. Despite intensive efforts to educate patients about effective oral hygiene procedures, ILs associated with fixed orthodontic appliances remain significant clinical problem. Factors such as the patient's medical history, dental history, medication history, diet, salivary flow rate, levels of calcium, phosphate, bicarbonate in saliva, fluoride levels, bracket type, and genetic susceptibility also play an important role $[5,7,8]$.

Many studies have focused on finding solutions for preventing ILs that occur during fixed orthodontic treatment [9-18]. Most studies in the literature specifically focused on dental materials used for bonding of orthodontic brackets [9-12] or for sealing the buccal surfaces of teeth $[13,14]$. Topical fluoride application of toothpastes, gels, rinses, varnishes, and sealants was found to be beneficial in patients with fixed appliances [15-17]. Chlorhexidine varnish treatment was found to be beneficial in inhibiting salivary mutans streptococci (MS) levels and in reducing gingivitis, therefore importing oral hygiene in these patients [18]. However, these treatments require patient compliance [19].

Citation: Karabekiroglu S, Erdur EA, Ileri Z, Unlu N (2018) Effectiveness of Remineralization Treatments on Incipient Lesions: A Controlled Clinical Trial. Int J Oral Dent Health 4:054. doi.org/10.23937/24695734/1510054

Accepted: June 11, 2018: Published: June 13, 2018

Copyright: (C) 2018 Karabekiroglu S, et al. This is an open-access article distributed under the terms of the Creative Commons Attribution License, which permits unrestricted use, distribution, and reproduction in any medium, provided the original author and source are credited. 
Minimally invasive paste, a new remineralization product, contains casein phosphopeptide-amorphous calcium phosphate (CPP-ACP). This active ingredient has been shown in many publications to be successful in the reduction of ILs. The active agent CPP-ACP is thought to stabilize and localize calcium, fluoride, and phosphate at the tooth surface in a slow-release amorphous form, thus enhancing the deeper remineralization of ILs [20]. CPP-ACP has been demonstrated to have anticariogenic potential in laboratory, animal, and human in situ experiments [21]. Many studies reported the effect of CPP-ACP usage on regression of initial labial/buccal surface lesions after orthodontic treatment [22]. On the other hand, only two studies $[23,24]$ investigated the remineralization effect of CPP-ACP paste versus twice-daily brushing with fluoride toothpaste during orthodontic treatment. Another study examined the effect of CPP-ACP on occlusal incipient lesions using Diagnodent [25]. The literature research showed that the duration of the follow-up was too short (4-12 weeks) in general, and the extended one lasted for 24 weeks. CPP-ACP products are much more costly than fluoride methods, so it is important to question the proof sustaining their common usage.

Early detection of ILs during orthodontic treatment is of great importance as it would allow clinicians to implement preventive measures for controlling the demineralization process before lesions progress. Some reviews of the literature showed no comparative studies on the different preventive methods for treating ILs during orthodontic treatment $[17,21,22]$. Several studies compared the effect of CPP-ACP to other products (fluoride gel, varnish, toothpaste, mouth rinse, or chlorhexidine gel) and methods with or without a control group [22]. Therefore, the objective of this study was to compare the effectiveness of four different methods (fluoride gel, fluoride varnish, chlorhexidine varnish, and (PP-ACP paste) in treating ILs during orthodontic treatment with a fixed appliance. The null hypothesis to be tested was that no statistically significant difference exists between the control and treatment groups.

\section{Materials and Methods}

The patients and their parents were informed about the clinical study and signed informed consent forms. The study protocol was approved by the School of Dentistry Ethics Committee at the University of Selçuk (2010-04/16).

\section{Trial design and subjects}

This study was a randomized, single-blind, four-parallel-group, and controlled clinical trial. A power analysis was established by $\mathrm{G}^{*}$ Power software (Ver. 3.0.10; Franz Faul, Universitat Kiel, Germany). A total sample size of 188 teeth (47 teeth per group) would give more than $95 \%$ power to detect significant differences with a 0.25 effect size among four groups and at a $p=0.05$ significance level.
Fifty-eight participants (from 12 to 17-years-old) who were undergoing routine orthodontic treatment were recruited for this prospective clinical study. The following inclusion criteria were applied: Had undergone no previous fixed orthodontic treatment, had permanent dentition, was in the first two months of fixed appliance therapy, had all anterior teeth fully erupted and fully visible at the start of the study, had all anterior teeth bonded, and did not smoke. The exclusion criteria were patients who: had systemic problems; had restorations or cavities in any anterior teeth; Had enamel hypoplasia, dental fluorosis, or intrinsic and extrinsic pigmentation; had plaque accumulation and a periodontal problem; had been taking antibiotics or any drugs during the past three months; had high caries activity (such as low saliva secretion rates or high DMFT value in the posterior region) or extensive caries in the posterior teeth; had been taking probiotic supplements during the past three months; had an allergy to IgE casein; or Regularly used fluoride mouthwash.

All patients were treated with 0.018-inch slot MBT fixed orthodontic appliances (Equilibrium ${ }^{\circledR} 2$, Dentaurum, Germany), and their teeth were bonded with a light-cured composite resin and adhesive (Transbond XT; 3M Unitek, Monrovia, Calif, USA). At the beginning of the treatment and all recalls, patients were instructed on tooth brushing in practice on a plastic tooth model. Patients were then asked to practice in front of a mirror. The WHO criteria were utilized to diagnose the carious status of the subjects [26]. Clinical examination was conducted by one examiner using a plane mouth mirror and blunt sickle probe with the aid of a dental chair light on dried teeth. Proximal regions were evaluated on a bitewing radiograph, which was not older than three months. The fluoride level of drinking water is less than $0.3 \mathrm{ppm}$ in Turkey. The digital bitewing images were obtained using the same intraoral unit (Trophy CCX Digital periapical $X$-ray Machine, France) using number 2 Digora phosphor plates at $65 \mathrm{kV}, 8 \mathrm{~mA}$. After the plates were exposed, they were processed by Sorodex Digora Optime, France. All proximal surfaces can be observed clearly in bitewings. All teeth that had carious lesions were restored with amalgam or resin composite before bracket bonding.

The anterior teeth of the subjects who met the criteria of inclusion were evaluated according to the IL index [5] with the help of standard loupes (3.0x Keeler Corporation, England). The labial surfaces of the upper and lower anterior teeth were scored as: $0=$ No white spot formation (No IL), 1 = Slight white spot formation (Slight IL), 2 = Excessive white spot formation (Severe IL), and $3=$ White spot formation with cavity formation (Cavity). Subjects without any sign of IL on any anterior teeth were excluded from the study $(\mathrm{N}=21)$. According to the index classification: (a) The teeth were restored that showed signs of cavities $(n=4)$, (b) The teeth without any signs of ILs were not assessed with DD $(n=105)$, and (c) Teeth that showed slight and severe ILs were 
evaluated with DD at baseline $(n=239)$. Afterward, only teeth that obtained a $D D$ value greater than zero were included in the randomization $(n=218)$.

The patients were randomized using an Internet-based computer program. Finally, these subjects were divided into four groups $(N=29)$ : The control group, the fluoride varnish (FL-V) group, the chlorhexidine varnish ( $\mathrm{CHX}-\mathrm{V}$ ) group, and the CPP-ACP group. A caries risk assessment was used to determine the caries risk of all subjects. The P-DMFT index (decay + missing + filled posterior teeth number) and P-DMFS index (decay + missing + filled posterior teeth surface number) were recorded at TO. DMFT and DMFS parameters were not evaluated at other visits because orthodontic brackets made radiographic evaluation difficult.

\section{Control group}

The subjects of all the groups were well educated and motivated to use the fluoride toothpaste (Colgate Total, $1.450 \mathrm{ppm}$ F) two times a day during the study period. All toothpastes were provided by the researchers to ensure consistency. For control group subjects, the following procedures were performed at each 3-month appointment for 6 months (intervention period) by the same operator: Oral hygiene instructions, professional tooth brushing of the IL areas with fluoride toothpaste for at least 3 minutes, and topical application of a 1.23\% APF gel (Topex gel, Sultan Dental Products, Englewood, $\mathrm{NJ}$ ) according to the manufacturer's instructions. Fluoride gel applications were applied just after the baseline DD readings and at $3^{\text {rd }}$ and $6^{\text {th }}$ month follow-up visits.

\section{Fluoride varnish group}

The subjects in this group received Enamel Pro-Varnish (Premier Dental, PA, USA) applications just after the baseline DD readings and at $3^{\text {rd }}$ and $6^{\text {th }}$ month follow-up visits. Topical application of $5 \%$ sodium fluoride varnish was applied according to the manufacturer's instructions and allowed to dry for 5 minutes. Subjects were warned not to eat/drink or brush/floss for 4-6 hours after the application of varnish. The following day, subjects resumed their normal oral hygiene regimen.

\section{Chlorhexidine varnish group}

The subjects received a Cervitec Plus varnish (1\% chlorhexidine diacetate and $1 \%$ thymol, Cervitec, IvoclarVivadent, Schaan, Liechtenstein) application for all teeth just after the baseline DD readings and at $3^{\text {rd }}$ and $6^{\text {th }}$ month follow-up visits. Products were conducted according to the manufacturer's instructions. After the varnish application, the varnish was dispersed with air and allowed to dry, and the cotton rolls were removed after 30 seconds. The subjects were informed not to rinse, eat/drink, or brush/floss for one hour.

\section{CPP-ACP group}

The subjects of the CPP-ACP group were instructed to locally apply a standardized amount of a CPP-ACP cream (Tooth Mousse, GC Europe N. V., Leuven, Belgium) to the teeth twice daily for a period of six months after the baseline DD readings [27]. The CPP-ACP paste was left undisturbed on the teeth. Participants were asked to keep their mouths open for at least 4 minutes after applying the agent. The subjects were also asked to expectorate and not to rinse their mouths, eat, or drink after using the paste. The subjects were instructed to brush their teeth with standard fluoride toothpaste (Colgate Total, $1.450 \mathrm{ppm}$ F) twice a day before CPP-ACP applications. No side effects or safety concerns were reported by any of the subjects in the use of all remineralization methods.

\section{Measurements}

At baseline (T0), after the intervention period (T1), and at the 12-month period (T2), measurements of the IL of each anterior tooth (the same site tested at each appointment) in both arches were taken with a Diagnodent pen (2190, KaVo, Biberach, Germany). Prior to its use, the instrument was calibrated in accordance with the manufacturer's instructions, and it was recalibrated for each examination session for each subject. All anterior teeth were cleaned and dried using an air syringe before the DD was used under cotton roll isolation. The angle of the tip was rotated and scanned over the area of interest on the labial tooth surface to record
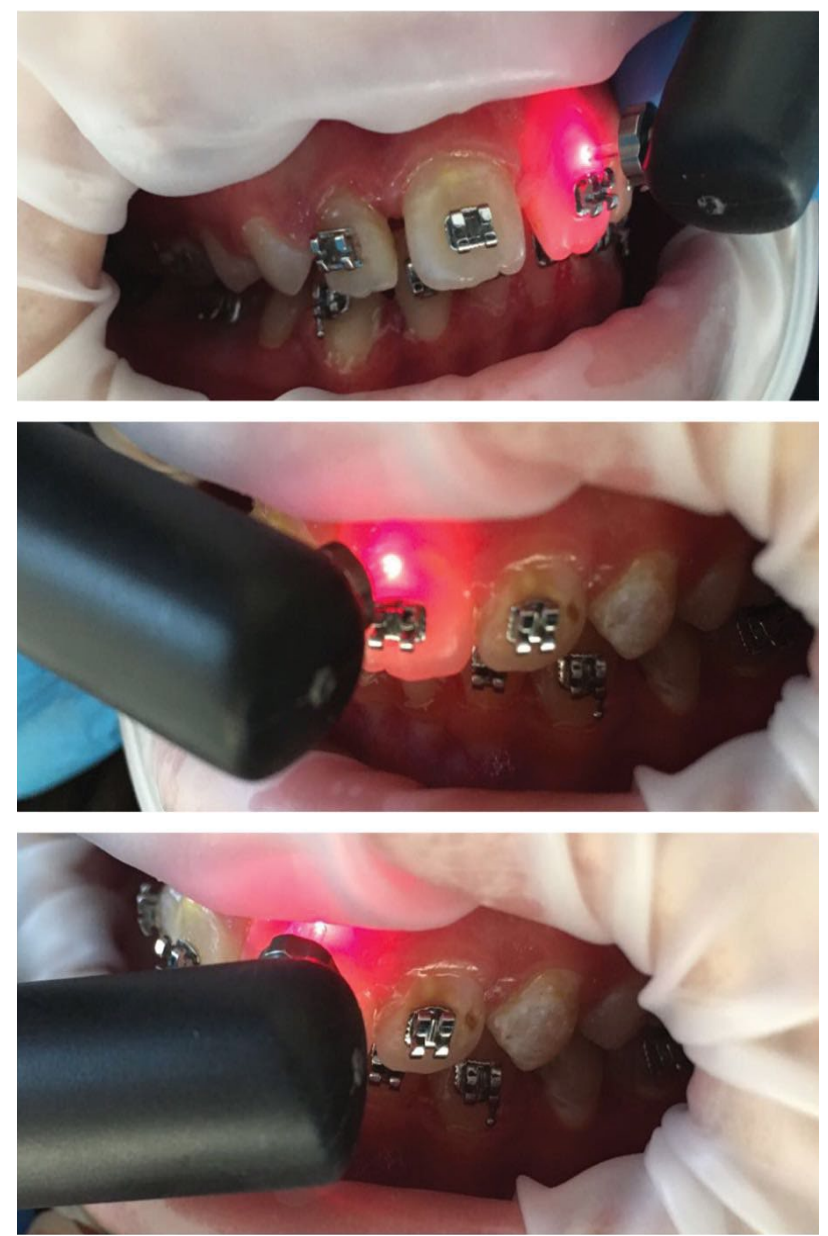

Figure 1: The application of DD readings. 


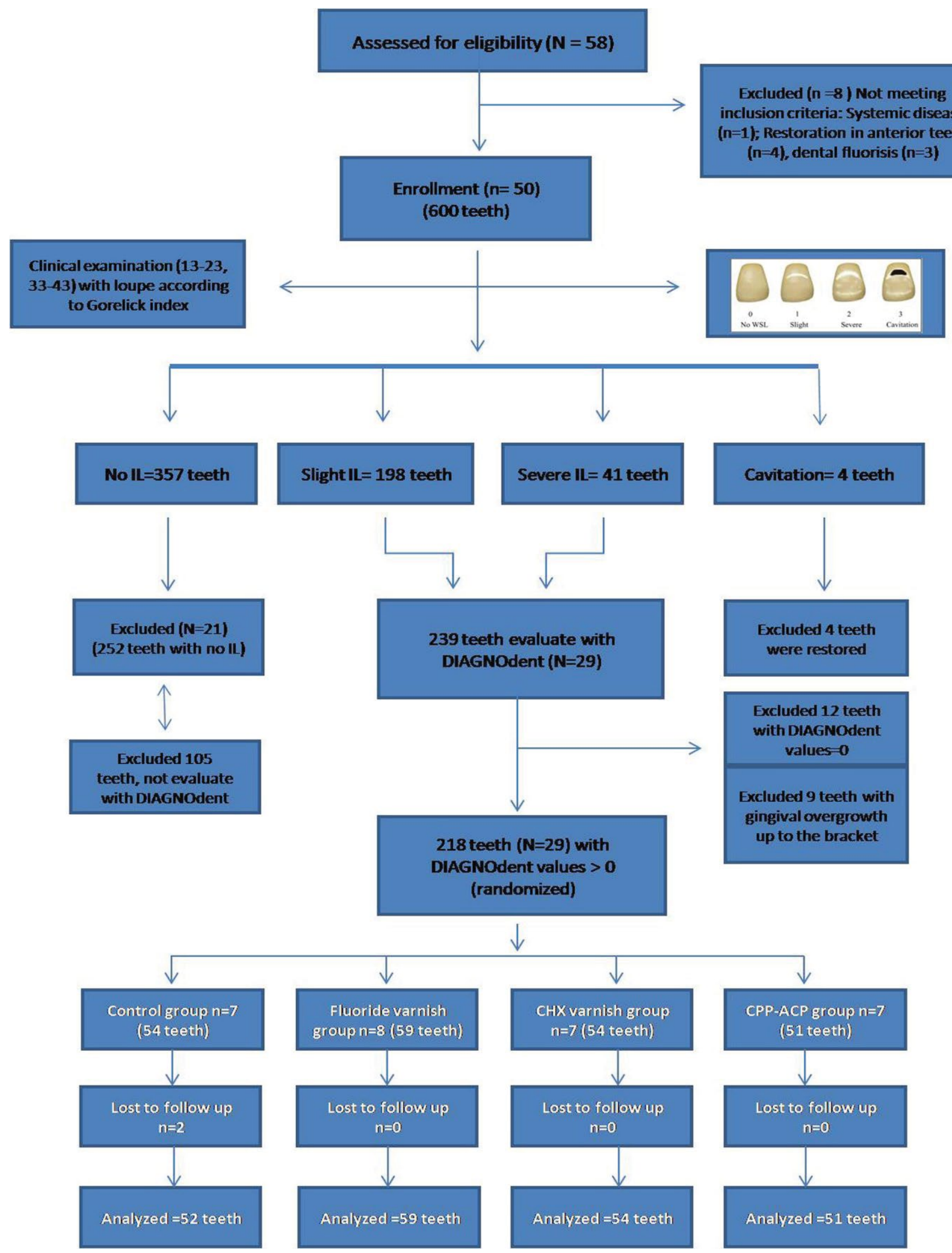

Figure 2: The schematic design of the study.

the peak value, which could range from 0 (sound) to 99 (caries). The results of the three measurements (which were performed in triplicate positions) were then added together and divided by three. The manufacturer suggested the presence and depth of a caries lesion at the smooth surfaces by DD with this scale: $0-13=$ Initial caries lesion; $14-20=$ Enamel caries; $21-30=$ Initial dentine caries; 31-99 = Advanced dentine caries (Clinical Guidelines, Kavo; 2002). The evaluation of ILs with DD during all visits was carried out by the same operator who was blind as to the group allocation of the subjects (Figure 1).

The severity status of IL was recorded at each of 
Table 1: Study subjects and number of teeth for each group.

\begin{tabular}{|l|l|l|l|l|l|l|}
\hline & Male & Female & Age & Teeth & P-DMFT & P-DMFS \\
\hline Control (FL-gel) & 3 & 4 & $14.8 \pm 2.0$ & 52 & 2.16 & 2.83 \\
\hline FL-V & 3 & 5 & $14.7 \pm 1.8$ & 59 & 2.28 & 3.14 \\
\hline CHX-V & 3 & 4 & $14.8 \pm 1.7$ & 54 & 1.83 & 2.5 \\
\hline CPP-ACP & 4 & 3 & $15.1 \pm 2.2$ & 51 & 2.66 & 3.16 \\
\hline
\end{tabular}

Table 2: Comparison of scores by DD $(x \pm S D)$ between groups.

\begin{tabular}{|l|l|l|l|}
\hline $\begin{array}{l}\text { DD readings } \\
\text { Groups }\end{array}$ & $\begin{array}{l}\text { Baseline } \\
\text { Mean } \pm \text { SD }(\text { min-max })\end{array}$ & $\begin{array}{l}\mathbf{1 2} \text { month } \\
\text { Mean } \pm \text { SD (min-max) }\end{array}$ & \begin{tabular}{l} 
Mean \pm SD (min-max $)$ \\
\hline Control (FL-gel)
\end{tabular} \\
\hline FL-V & $3.30 \pm 1.66^{\mathrm{a}}(1-8)$ & $3.61 \pm 2.47^{\mathrm{c}}(0-12)$ & $4.30 \pm 3.35^{\mathrm{d}}(0-14)$ \\
\hline CHX-V & $3.50 \pm 2.41^{\mathrm{a}}(1-11)$ & $3.33 \pm 2.30^{\mathrm{a}}(0-10)$ & $3.83 \pm 2.91^{\mathrm{c}}(0-12)$ \\
\hline CPP-ACP & $3.55 \pm 1.94^{\mathrm{a}}(1-8)$ & $3.05 \pm 1.78^{\mathrm{a}}(0-6)$ & $3.66 \pm 2.94^{\mathrm{c}}(0-13)$ \\
\hline
\end{tabular}

Means with the same superscript letter are not statistically different from each other $(p>0.05)$.

three points. The ICDAS II criteria were used with the aid of loupes for visual evaluation of the labial surfaces. Only modified ICDAS II codes 1,2 , and 3 were included in the study [27]. ICDAS II codes 4,5 , and 6 were not included in the analysis since any teeth with these scores were excluded from the study. Scoring was performed under direct illumination using a dental lamp after light pumicing. A visual inspection of labial tooth surfaces for IL was undertaken with tooth surfaces initially wet and again after 5 seconds of drying with a gentle air stream. Measurements were taken by the same operator for all subjects who were enrolled in the study. To assess the reproducibility of the diagnostic criteria application, an intra-examiner calibration was performed. An intra-examiner test was conducted by re-examining 20 randomly selected teeth one week after the first examination. The level of intra-examiner agreement was measured using Cohen's kappa statistics. The intra-examiner agreement for caries detection was good, with a Kappa value of $85 \%$. The progression or regression status of each lesion was based on a labial surface comparison between the time points. The following scores were given: Score 0 (unchanged-stable IL); Score + (improved-regressed IL); and Score - (worsened-progressed IL). Each score was also recorded for lesions changing from code 1-2 to code $0-1$ or vice versa.

\section{Statistical analysis}

The statistical analysis was completed with the SPSS 17.0 software system (SPSS Inc., Chicago, Illinois, USA). A p-value of $<0.05$ was accepted as being statistically significant. Descriptive statistics, including the means, standard deviations, and frequencies (percentages), were calculated. Because the results of the Kolmogorov-Smirnov and Shapiro-Wilk tests showed that the data were not normally distributed ( $p<0.05$ ), nonparametric tests were used for statistical analysis. Therefore, the data were analyzed by the Kruskal-Wallis test. The Mann-Whitney $U$ test was applied to determine specific differences between the groups. The Friedman test was used to evaluate the repeated measures. For all ILs, the progression or regression scores were analyzed by means of a proportional odds ordinal logistic regression model. The worsened and unchanged lesions were combined, and improved ILs was defined as the positive outcome group. The combined counts were then analyzed by a logistic regression model.

\section{Results}

A total of 50 subjects met the inclusion criteria for participation in this study (Figure 2). A total of 600 teeth were evaluated according to IL index. However, 218 teeth that could obtain DD $>0$ were included in the randomization. The study subjects, age, teeth, and P-DMFT and P-DMFS means are shown in Table 1.

The ILs had a mean DD reading at baseline of $3.30 \pm$ 1.66 in the control group, $3.50 \pm 2.41$ in the FL-V group, $3.55 \pm 1.94$ in the $\mathrm{CHX}-\mathrm{V}$ group, and $3.43 \pm 1.75$ in the CPP-ACP group. There were no significant differences between the four groups at baseline $(p>0.05)$. Statistically significant differences were found between the mean DD readings of the control and CPP-ACP groups $(p=0.002), F L-V$ and CPP-ACP groups $(p=0.035)$, and CHX-V and CPP-ACP groups ( $p=0.017)$ at T1. The DD values of the ILs decreased in all groups in the intervention period (T0-T1) except for the control group (Table $2)$. The mean DD readings increased in all groups during the follow-up period (T1-T2). A statistically significant difference was found between the CPP-ACP and other groups at T2. The control group showed the highest DD increment between the $6^{\text {th }}$ and $12^{\text {th }}$ months.

The ICDAS II scores of each group are shown in Table 3. The percentages of ILs that were treated during the study period for all groups are displayed in Table 4 . The differences in the improvement score distributions between the four treatment groups were not statistically significant $(p>0.05)$. According to the results, more lesions were regressed in the $\mathrm{CHX}-\mathrm{V}$ and $\mathrm{CPP}-\mathrm{ACP}$ groups during the first 6 months, but these were not statistically different from the control and FL-V groups ( $p>0.05)$. For ILs, $10 \%$ more had treated with the CPP-ACP cream than with the control group (FL-gel) during the first 6 -month period. Most of the lesions were stable during 
the 6 and 12 months. There were no statistically significant differences between all test groups for ICDAS II scores during the follow-up period $(p>0.05)$. All groups had lower improvement scores during the second period than during the intervention period (Table 4).

\section{Discussion}

This study was carried out to determine which remineralization method is the most successful in treating ILs during orthodontic treatment with a fixed appliance. The preventive agents employed were fluoride gel (control), fluoride varnish, chlorhexidine varnish, and CPPACP paste. The efficacy of the different groups was evaluated with a DIAGNOdent pen at three different times during 12 months. The null hypothesis was partially rejected; It was found that CPP-ACP is not better than other methods for improving the appearance of initial lesions after 6 months of use, but it is more effective for treating subsurface ILs according to DD results.

\section{Clinical problem}

Individuals with malocclusion have many plaque-retention areas due to tooth alignment-related defects. It

Table 3: Number and percentage of lesions according to ICDAS II criteria.

\begin{tabular}{|c|c|c|c|c|}
\hline Groups & $\begin{array}{l}\text { ICDAS II } \\
\text { Codes }\end{array}$ & Baseline & 6 month & 12 month \\
\hline \multirow{4}{*}{ Control (FL-gel) } & 0 & - & 0 & 0 \\
\hline & 1 & $12(23.0)$ & $17(32.6)$ & $16(30.7)$ \\
\hline & 2 & $32(61.5)$ & $31(59.6)$ & $33(63.4)$ \\
\hline & 3 & $10(19.2)$ & $6(11.5)$ & $5(9.6)$ \\
\hline \multirow{4}{*}{ FL-V } & 0 & - & $3(5.0)$ & $4(6.7)$ \\
\hline & 1 & $16(27.1)$ & $24(40.6)$ & $22(37.2)$ \\
\hline & 2 & $35(59.3)$ & $28(47.4)$ & $27(45.7)$ \\
\hline & 3 & $8(13.5)$ & $4(6.7)$ & $6(10.1)$ \\
\hline \multirow{4}{*}{ CHX-V } & 0 & - & $2(3.7)$ & $3(5.5)$ \\
\hline & 1 & $14(25.9)$ & $23(42.5)$ & $26(48.1)$ \\
\hline & 2 & $37(68.5)$ & $29(53.7)$ & $23(42.5)$ \\
\hline & 3 & $3(5.5)$ & 0 & $2(3.7)$ \\
\hline \multirow{4}{*}{ CPP-ACP } & 0 & - & $5(9.8)$ & $4(7.8)$ \\
\hline & 1 & $10(19.6)$ & $14(27.4)$ & $16(31.3)$ \\
\hline & 2 & $34(66.6)$ & $30(58.8)$ & $29(56.8)$ \\
\hline & 3 & 7 (13.7) & $2(3.9)$ & $2(3.9)$ \\
\hline
\end{tabular}

has been shown that there is a significant increase in the prevalence and severity of enamel demineralization after orthodontic treatment when compared with untreated control subjects [2,4]. Appearance of these lesions results in problems such as poor aesthetics and patient dissatisfaction after the orthodontic treatment. Due to these problems, it is important to determine the saliva, oral care status, and caries risk of the patient and, if necessary, evaluate the initial preventive applications before the orthodontic treatment [19]. Based on the caries risk of the individual, ILs may progress and form cavities, may remain for a long time without progressing, or may heal if they are at a reversible level. On the other hand, an accurate evaluation of demineralized ILs during orthodontic treatment is important, so early prevention and/or treatment should be performed [19].

\section{Different remineralization methods}

Routine twice-daily tooth brushing is proposed by many clinicians as an important part of a daily oral hygiene and plaque control program for all orthodontic patients [10]. The additional use of fluoride, chlorhexidine varnish, and CPP-ACP products can be effective in remineralizing demineralized enamel [28]. Scientifically, the caries-preventive effect of fluoride is explained by the fact that fluoride can be integrated into the crystal structure of dental enamel to create a more resistant structure against acid [29]. During orthodontic treatment, fluoride can be applied to the teeth in various ways, including by topical (fluoridated toothpaste, mouth rinse, gel, and varnish) and adhesive (fluoride-releasing cements and elastomeric modules and chains) methods [17]. Chlorhexidine varnish used as an addition to fluoride therapy has been proven to have demineralization-inhibiting effects in patients with fixed orthodontic appliances [30]. The role of CPP-ACP in decreasing the incidence of ILs in the orthodontic population is expected to be additive to the beneficial effects of fluoridated toothpaste [28].

\section{Fluoride and chlorhexidine groups}

The results of this study showed that when fluoride or chlorhexidine varnishes were applied, laser fluorescence reading reduction of ILs was significantly different from that in the fluoride gel group. Fluoride varnish was

Table 4: Frequency distributions of incipient lesion transitions during the study periods $(\uparrow=$ worsened, $\leftrightarrow=$ unchanged, $\downarrow=$ improved $)$.

\begin{tabular}{|l|l|l|l|l|l|l|}
\hline Baseline-6 month & $\uparrow$ & $\leftrightarrow$ & $\downarrow$ & OR $^{\mathbf{a}}$ & $\mathbf{9 5 \%} \mathbf{C l}$ & \\
\hline Control (FL-gel) & $3(5.7)$ & $39(75.0)$ & $12(23.0)$ & & & \\
\hline FL-V & $2(3.3)$ & $41(69.4)$ & $16(27.1)$ & 1.23 & $0.65-2.35$ & 0.514 \\
\hline CHX-V & $2(3.7)$ & $34(52.9)$ & $18(33.3)$ & 1.64 & $0.88-3.08$ & 0.116 \\
\hline CPP-ACP & $3(5.8)$ & $31(60.7)$ & $17(33.3)$ & 1.64 & $0.88-3.08$ & 0.116 \\
\hline 6 month-12 month & $\uparrow$ & $\leftrightarrow$ & $\downarrow$ & $\mathrm{OR}^{\mathrm{a}}$ & $95 \% \mathrm{Cl}$ & $\mathrm{p}$ \\
\hline Control (FL-gel) & $3(5.7)$ & $45(86.5)$ & $3(5.7)$ & & & \\
\hline FL-V & $5(8.4)$ & $51(86.4)$ & $3(5.0)$ & 0.82 & $0.24-2.79$ & 0.756 \\
\hline CHX-V & $3(5.5)$ & $46(85.1)$ & $5(8.4)$ & 1.36 & $0.45-4.07$ & 0.58 \\
\hline CPP-ACP & $4(7.8)$ & $43(84.3)$ & $4(7.8)$ & 1.17 & $0.38-3.64$ & 0.774 \\
\hline
\end{tabular}

aThe odds of lesion regressing with FL-V, CHX-V, CPP-ACP compared with the odds of lesion regressing with fluoride gel. 
found to be less effective than chlorhexidine varnish, but it was not significantly different. Clinical studies investigating the action of a fluoride or chlorhexidine program in orthodontic patients have shown contradictory results $[22,23,30,31]$. One study in the literature investigated two varnish materials together [30]. It has been suggested that chlorhexidine application in the form of a varnish results in longer-lasting suppression of MS concentrations compared with other forms of application. Øgaard, et al. [30] reported a clear trend that the combination of antimicrobial and fluoride varnishes more effectively reduced the increments of new ILs compared with fluoride varnish alone. It has been reported that the application of a fluoride varnish resulted in a $44.3 \%$ reduction in enamel demineralization in orthodontic patients [31]. Perrini, et al. [32] reported that periodic administration of fluoride varnish can present remineralization against initial lesions at labial surfaces, but not to a statistically significant level if the patients have excellent oral care. Attin, et al. [33] reported that chlorhexidine varnish reduced salivary $M S$ significantly over four weeks compared to baseline values in orthodontic patients. The variation of different results among studies could be attributed to differences in the number of teeth (or subjects) examined, the examination methods (visual or fluorescent), the location of the study sample (cultural or socioeconomic differences), the past caries experience, treatment or follow-up duration, and materials.

\section{CPP-ACP group}

Use of the CPP-ACP-containing products is thought to be effective in preventing and treating ILs [20]. Reynolds, et al. [21] stated that CPP-ACP buffers the free calcium phosphate ion activity in the form of amorphous calcium phosphate accumulated on the tooth surface and provides the super saturation of the enamel, thus preventing demineralization. In a limited number of studies, CPP-ACP-containing products were used on individuals undergoing orthodontic treatment, and ILS were examined $[23,24]$. Robertson, et al. [24] reported that CPP-ACP not only resulted in the remineralization of caries lesions during orthodontic treatment but also decreased the number of ILs. According to the results of our study, CPP-ACP was found to have had the same effect on the appearance of ILs compared to the other groups. In the study by Robertson, et al. [24], the length of the follow-up was three months, visual evaluation methods were used, and the CPP-ACP application type and frequency were different from those of our study.

Another study, conducted by Wang, et al. [23], investigated the remineralization potential of CPP-ACP for enamel decalcification and reported that CPP-ACP can effectively improve the demineralized initial lesions during orthodontic treatment. On the other hand, our study results should be read carefully because although a difference was found between the groups in terms of laser fluorescence averages, no clinically significant difference was found in the visual evaluation. There were many limitations to follow-up in the clinical studies [24,34]. First of all, similar compliance of all of the individuals, their standardization, or keeping them under control was difficult. Therefore, individuals who had irregular oral care or high risk for dental caries were not included in our study. These factors could affect our study results.

\section{Monitoring of incipient lesions}

Photographic examination, clinical examination, optical non-fluorescence methods, and optical fluorescence methods were used in the diagnosis of ILs [35]. We considered that visual inspection with the Gorelick index after air drying and tactile examination by dental probing was appropriate in the present study at first evaluation. After that, tooth surfaces with ILs were assessed by a DD, and the readings, which estimated the organic content and bacterial metabolites in caries lesions, were used to indicate the changes of the ILs. DIAGNOdent had acceptable sensitivity and specificity when compared to QLF. DIAGNOdent had some disadvantages, such as the fact that readings could also be affected by stains, calculus, and plaque and were based on bacterial metabolites [35]. Assessment of biological and cosmetic changing of ILs was the focus in this study, so laser fluorescence and ICDAS II were used together. Bailey, et al. reported that ICDAS II allows for the detection of dental caries at the initial grade based on visual estimation of the enamel surface and has been presented to have substance and correlational currency with histological lesion depth. Most of the lesions in our study were ICDAS II code 1 and 2, and code 1 is probably the most challenging classification to make [27]. These situations may have affected our results between the test groups. The evaluation of the caries severity status of initial lesions is currently very challenging as it depends on the investigator's ability to define minor changes in enamel by visual and tactile inspection.

\section{Follow-up periods}

In the second part of our study (T1-T2), preventive applications were not performed for the individuals, but a six-month follow-up was performed. Elevation was seen in the laser fluorescence averages of all of the groups during this period. In this second six-month period, the remineralized tooth rate was similar in all groups. On the other hand, when laser fluorescence averages were examined, the minimum elevation was in the CPP-ACP group, while the maximum elevation was in the fluoride gel group. Accordingly, it can be considered that CPP-ACP-applied teeth are more resistant to subsequent the next demineralization attacks. The presence of CPP-ACP might permit a serial proceed to resting calcium concentrations and empowered earlier remineralization of enamel subsurface. According to our results, fluoride varnish was found to be more effec- 
tive than gel formulation for prevention of subsurface ILs during the follow-up period. It is well known that if fluoride is present in the plaque fluid, it will travel with the acid into the subsurface region, where it adsorbs to the surface of the crystals, protecting them against dissolution. Fluoride varnish attended to the enamel surface longer than other topical fluoride methods and has been shown to be superior to the use of sodium fluoride and monofluorophosphate toothpastes and acidulated phosphate fluoride gel application because of its ability to increase fluoride uptake in enamel in vitro [36]. On the other hand, the different results between gel $(12,500 \mathrm{ppm} F)$ and varnish $(22,500 \mathrm{ppm} F)$ groups in our study could be related to the difference in fluoride concentration.

It has been demonstrated that chlorhexidine application in the form of a varnish results in longer-lasting suppression of bacteria levels compared with other forms of application. The results of this study showed that chlorhexidine varnish application was as effective as CPP-ACP cream between the $6^{\text {th }}$ and $12^{\text {th }}$ month in terms of lesion appearance. It should be considered that the decrease in the motivation of the individual could have contributed to these results. Finally, in the individuals undergoing fixed orthodontic treatment, before starting the treatment, general caries risk evaluation should be performed, and patients should be categorized according to caries risk indication. In addition, it should be remembered that a majority of patients undergoing fixed orthodontic treatment are at low ages and have little motivation. An additional preventive application should absolutely be performed for individuals determined to have poor oral care habits. Thus, ILs that may appear at the end of the treatment can still be treated in the mild stage.

\section{Conclusions}

The following results can be drawn from this randomized controlled trial;

1. The daily usage of CPP-ACP was as effective as other test methods for improving the appearance of ILs, and it was found to significantly increase the remineralization of subsurface lesions.

2. Fluoride gel (control group) application was less effective than fluoride varnish, chlorhexidine varnish and CPP-ACP for regression of subsurface ILs during orthodontic treatment with fixed appliance.

\section{References}

1. Ogaard B, Bishara S, Duschner H (2004) Enamel effects during bonding-debonding and treatment with fixed appliances. In: Graber TM, Eliades T, Athanasiou A, (1 st $^{\text {edn }}$ ), Risk Management in Orthodontics: Experts' Guide to Malpractice. Hanover Park, IL: Quintessence Publishing, 1946.

2. Ogaard B, Rølla G, Arends J (1988) Orthodontic appliances and enamel demineralization. Part 1. Lesion development.
Am J Orthod Dentofacial Orthop 94: 68-73.

3. Boersma JG, van der Veen $\mathrm{MH}$, Lagerweij MD, Bokhout B, Prahl-Andersen B (2005) Caries prevalence measured with QLF after treatment with fixed orthodontic appliances: Influencing factors. Caries Res 39: 41-47.

4. Uysal T, Amasyali M, Koyuturk AE, Ozcan S (2010) Effects of different topical agents on enamel demineralization around orthodontic brackets: An in vivo and in vitro study. Aust Dent J 55: 268-274.

5. Akin M, Tezcan M, lleri Z, Ayhan F (2015) Incidence of white spot lesions among patients treated with self- and conventional ligation systems. Clin Oral Investig 19: 15011506.

6. Akin M, Tezcan M, lleri Z, Basciftci FA (2013) Incidence of white spot lesion during fixed orthodontic treatment. Turkish J Orthod 6: 98-102.

7. Chalmers JM (2006) Minimal intervention dentistry: Strategies for the new caries challenge in our older patients. JCDA 72: 325-331.

8. Papas AS, Joshi A, MacDonald SL, Maravelis-Splagounias L, Pretara-Spanedda P, et al. (1993) Caries prevalence in xerostomic individuals. J Can Dent Assoc 59: 171-179.

9. Anhoury $P$, Nathanson D, Hughes CV, Socransky S, Feres $M$, et al. (2002) Microbial profile on metallic and ceramic bracket materials. Angle Orthod 72: 338-343.

10. Bibby BG (1945) A test of the effect of fluoride-containing dentifrices on dental caries. J Dent Res 24: 297-303.

11. Paschos E, Kleinschrodt T, Clementino-Luedemann $T$, Huth KC, Hickel R, et al. (2009) Effect of different bonding agents on prevention of enamel demineralization around orthodontic brackets. Am J Orthod Dentofacial Orthop 135: 603-612.

12. Schmidlin PR, Schatzle M, Fischer J, Attin T (2008) Bonding of brackets using a caries-protective adhesive patch. J Dent 36: 125-129.

13. Benham AW, Campbell PM, Buschang PH (2009) Effectiveness of pit and fissure sealants in reducing white spot lesions during orthodontic treatment. A pilot study. Angle Orthod 79: 338-345.

14. Ghiz MA, Ngan P, Kao E, Martin C, Gunel E (2009) Effects of sealant and self-etching primer on enamel decalcification. Part II: An in-vivo study. Am J Orthod Dentofacial Orthop 135: 206-213.

15. Oh EJ, Park SS, Jang MJ, Jeon YM, Kim JG (2008) Experimental brush wear pattern and cariostatic effect of Biscover. Korean J Orthod 38: 214-222.

16. Jo SY, Chongb HJ, Lee EH, Chang NY, Chae JM, et al. (2014) Effects of various toothpastes on remineralization of white spot lesions. Korean J Orthod 44: 113-118.

17. Chadwick BL, Roy J, Knox J, Treasure ET (2005) The effect of topical fluorides on decalcification in patients with fixed orthodontic appliances: A systematic review. Am J Orthod Dentofacial Orthop 128: 601-606.

18. Øgaard B, Larsson E, Glans R, Henriksson T, Birkhed D (1997) Antimicrobial effect of a chlorhexidine-thymol varnish (Cervitec) in orthodontic patients. A prospective, randomized clinical trial. J Orofac Orthop 58: 206-213.

19. Ogaard B, Rolla G, Arends J, ten Cate JM (1988) Orthodontic appliances and enamel demineralization. Part II: prevention and treatment of lesions. Am J Orthod Dentofacial Orthop 94: 123-128. 
20. Reynolds EC (2008) Calcium phosphate-based remineralization systems: Scientific evidence? Aust Dent J 53: 268-273.

21. Reynolds EC (1998) Anticariogenic complexes of amorphous calcium phosphate stabilized by casein phosphopeptides: A review. Spec Care Dentist 18: 8-16.

22. Raphael S, Blinkhorn A (2015) Is there a place for Tooth Mousse $^{\circledR}$ in the prevention and treatment of early dental caries? A systematic review. BMC Oral Health 15: 113.

23. Wang JX, Yan Y, Wang XJ (2012) Clinical evaluation of remineralization potential of casein phosphopeptide amorphous calcium phosphate nanocomplexes for enamel decalcification in orthodontics. Chin Med J 125: 4018-4021.

24. Robertson MA, Kau CH, English JD, Lee RP, Powers J, et al. (2011) MI Paste plus to prevent demineralization in orthodontic patients: A prospective randomized controlled trial. Am J Orthod Dentofacial Orthop 140: 660-668.

25. Krithikadatta J, Fredrick C, Abarajithan M, Kandaswamy D (2013) Remineralisation of occlusal white spot lesion with a combination of $10 \%$ CPP-ACP and $0.2 \%$ sodium fluoride evaluated using Diagnodent: A pilot study. Oral Health Prev Dent 11: 191-196.

26. World Health Organization (1997) Oral health surveys, basic methods. ( $4^{\text {th }}$ edn), Geneva.

27. Bailey DL, Adams GG, Tsao CE, Hyslop A, Escobar K, et al. (2009) Regression of post-orthodontic lesions by a remineralizing cream. J Dent Res 88: 1148-1153.

28. Sudjalim TR, Woods MG, Manton DJ (2006) Prevention of white spot lesions in orthodontic practice: A contemporary review. Aust Dent J 51: 284-289.
29. ten Cate JM (1999) Current concepts on the theories of the mechanism of action of fluoride. Acta Odontol Scand 57: 325-329.

30. Ogaard B, Larsson E, Henriksson T, Birkhed D, Bishara SE (2001) Effects of combined application of antimicrobial and fluoride varnishes in orthodontic patients. Am J Orthod Dentofacial Orthop 20: 28-35.

31. Vivaldi-Rodrigues G, Demito CF, Bowman SJ, Ramos AL (2006) The effectiveness of a fluoride varnish in preventing the development of white spot lesions. World J Orthod 7: 138-144.

32. Perrini F, Lombardo L, Arreghini A, Medori S, Siciliani G (2016) Caries prevention during orthodontic treatment: In-vivo assessment of high-fluoride varnish to prevent white spot lesions. Am J Orthod Dentofacial Orthop 149: 238-243.

33. Attin R, Yetkiner E, Aykut-Yetkiner A, Knösel M, Attin T (2013) Effect of chlorhexidine varnish application on streptoococcus mutans colonisation in adolescents with fixed orthodontic appliances. Aust Orthod J 29: 52-57.

34. Chen H, Liu X, Dai J, Jiang Z, Guo T, et al. (2013) Effect of remineralizing agents on white spot lesions after orthodontic treatment: A systematic review. Am J Orthod Dentofacial Orthop 143: 376-382.

35. Benson $P$ (2008) Evaluation of white spot lesions on teeth with orthodontic brackets. Semin Orthod 14: 200-208.

36. Arends J, Lodding A, Petersson LG (1980) Fluoride uptake in enamel. In vitro comparison of topical agents. Caries Res 14: 403-413. 